J. Phys. IV France 130 (2005) 81-84

(C) EDP Sciences, Les Ulis

DOI: $10.1051 /$ jp4:2005130006

\title{
Mesures SANS sous saut de pression résolues en temps
}

\author{
M. Plazanet ${ }^{1}$, R. Schweins ${ }^{1}$, P. Lindner ${ }^{1}$ et H.P. Trommsdorff ${ }^{2}$ \\ ${ }^{1}$ Institut Laue Langevin, Grenoble, France \\ ${ }^{2}$ Laboratoire de Spectrométrie Physique, Grenoble, France
}

\section{INTRODUCTION}

La pression est une variable thermodynamique dont les effets sur le comportement des organismes sont nombreux: influence de la cinétique de réaction, cristallisation, dépliement des proteines, séparation de phase ou coagulation. Du point de vue de l'étude de la thermodynamique du système, la variation de température induit des effets dues à la variation de temperature et de densité, alors que la pression permet d'isoler les effets liés à la densité.

Dans les protéines, la réponse à une variation de pression ou de température est en effet nettement différente. Par exemple, certaines mutations peuvent entrainer un grand changement de réponse à la température et conserver le comportement sous pression exactement identique [1].

La figure 1 présente le diagramme de phase typique d'une protéine. Pour une pression maximale située autour de 5 à $6 \mathrm{kbars}$, la protéine subit un important dépliement (au delà du molten globule), sans toutefois être dénaturée. Il apparait clairement que lorsque la pression augmente, la compression du système force la pénétration de l'eau dans la protéine et donc l'exposition des parties hydrophobes au solvant, provoquant le dépliement. La protéine passe éventuellement par des états partiellement repliés, qui dépendent de la taille et la conformation de la protéines, et qui sont plus ou moins bien connus. Un état dit "molten globule", assez compact mais déja désordonné, a été reconnu dans un grand nombre de protéines.

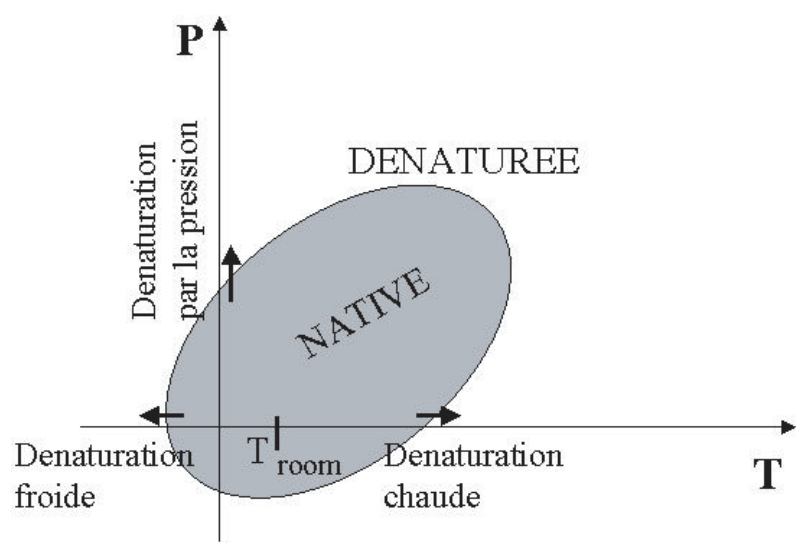

Figure 1. Représentation schematique du diagramme de phase elliptique des protéine.

Le diagramme de phase elliptique de la figure 1, similaire au diagramme d'une phase re-entrante, est valable pour de nombreuses protéines, mais pas dans toutes les macromolécules. En particulier, les bicouches lipidiques ou le DNA présentent un diagramme de phase droit [1]. 


\section{MESURES CINÉTIQUES}

Selon les protéines et les conditions expérimentales, la cinéti que de dépliement ou repliement varie de quelques microsecondes à quelques secondes. De nombreuses techniques expérimentales permettent de suivre la cinétique de dé/re-pliement faisant suite à la variation brusque d'une des conditions expérimentales (T, P, pH...). Les études cinétiques permettent de caractériser les états intermédiaires qui ne peuvent pas être piégés et étudiés de manière statique, et de déterminer la stabilité de ces états.

Pour illustrer la question, nous pouvons décrire une étude effectuée par diffusion de rayons X aux petits angles et fluorescence, résolus en temps, du repliement de la lysozyme [2]. En diluant rapidement une solution de lysozyme dénaturée, le $\mathrm{pH}$ passe brusquement de 1.8 à 5.2, permettant le repliement de la protéine. Quatre états ont été caractérisés par leurs rayons de gyration, ainsi que les constantes cinétiques de passage d'un état à l'autre à température ambiante. La figure 2, reproduite de la référence [2], illustre les quatre états : déplié (U), compact désordonné (C), intermédiaire lors duquel se forment les hélices (I), puis totalement replié $(\mathrm{N})$. Entre l'état $(\mathrm{C})$ et $(\mathrm{N})$, deux chemins de repliement ont été observées, attribués à la compétition entre la protection des parties hydrophobes, et la mise en place des contacts entre chaines latérales: dans le premier cas, le repliement se fait par un chemin direct, dans le second cas, la formation des helices est observée avant la forme native complète.

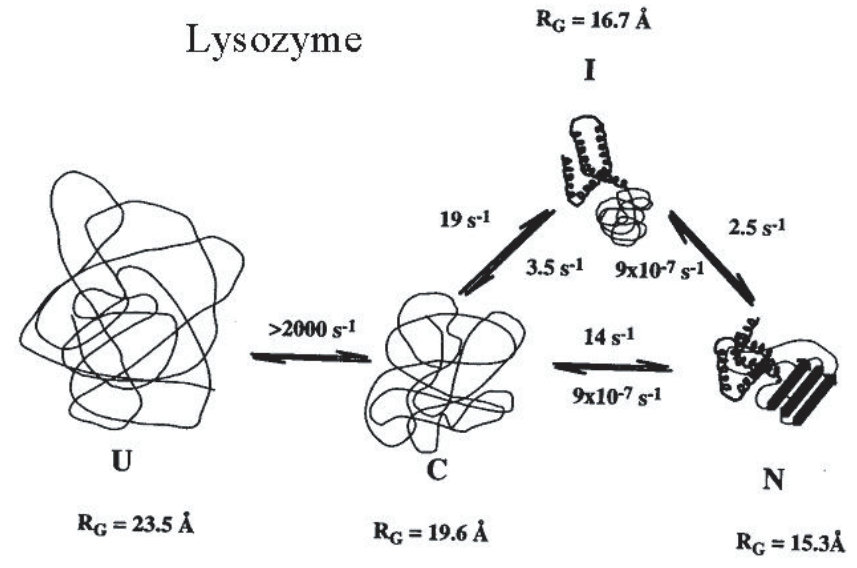

Figure 2. Reproduite de la réference 2: modèle cinétique pour les évènements suivant la dilution en $\mathrm{pH}=5.2(0.6 \mathrm{M}$ $\left.\mathrm{GdmCl}, 20^{\circ} \mathrm{C}\right)$, après laquelle la protéine se replie. Quatre états sont observées: déplié (U), fondu $(\mathrm{C})$, intermédiaire (I) et natif $(\mathrm{N})$.

Il est aussi interessant de décrire ici l'expérience faite par J. Woenckhaus et al. par SAXS suivant un saut de pression, présentée figure 3. Le montage expérimental permet d'effectuer un saut de pression de 1.5 à 3 kbars et d'observer le dépliement de la protéine, ainsi qu'un saut de 4.5 à 0.8 kbars en 5 ms afin d'observer le repliement. La protéine utilisée, Snase w.t., a un rayon de gyration qui varie d'environ 18 à $30 \AA$. Les deux cinétiques, repliement et dépliement, ont pu être caracterisées, présentant toutes deux une relaxation exponentielle, respectivement de 5 s et de 14 minutes. Une seule relaxation a éte mise en évidence, ne montrant pas l'existance d'état intermédiaire stable sur cette résolution temporelle.

\section{MONTAGE EXPÉRIMENTAL}

Le montage expérimental, développé à l'ILL, a été construit afin de réaliser des sauts de pression dans le temps le plus court possible. Le temps employé par le système pour pressuriser l'échantillon détermine la résolution temporelle avec laquelle le phénomène cinétique peut être étudié. Un tel montage a déja été réalisé pour être utilisé en SAXS [3]. 

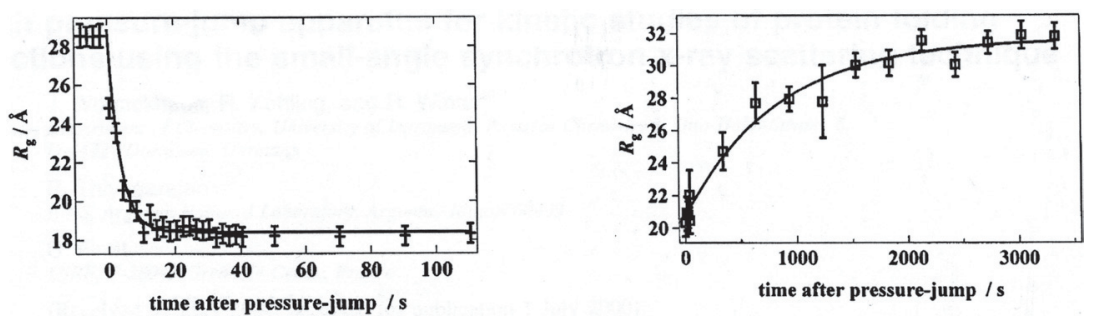

Figure 3. Expérience de J. Woenckhaus et al. [3] par SAXS suivant un saut de pression: à gauche, saut de 4.5 à $0.8 \mathrm{kbars}$ en $5 \mathrm{~ms}$, induisant le repliement de la Snase w.t. avec un temps caractéristique de $5 \mathrm{~s}$. A droite, saut de 1.5 à 3 kbars, induisant le dépliement de la protéine en 14 minutes.

Le montage est présenté sur la figure 4. Le circuit est composé de la pompe, la cellule pression, 2 vannes rapides, un séparateur de liquides et les capillaires reliant les éléments entre eux. La pression est initialement montée à pression Pi dans la première partie du circuit (pompe-vanne 1). La vanne 1 est brusquement ouverte pour induire le saut de pression dans la cellule : son temps de réaction détermine la rapidité du saut de pression. L'ensemble du circuit passe à la haute pression P. Après un certain temps de mesure, la pression est brusquement relachée à une valeur $\mathrm{Pb}$ en ouvrant la vanne 2 , ce qui engendre le saut de pression dans l'autre sens. Après le temps de mesure, la partie amont du circuit est re-pressurisée et un second cycle peut débuter.

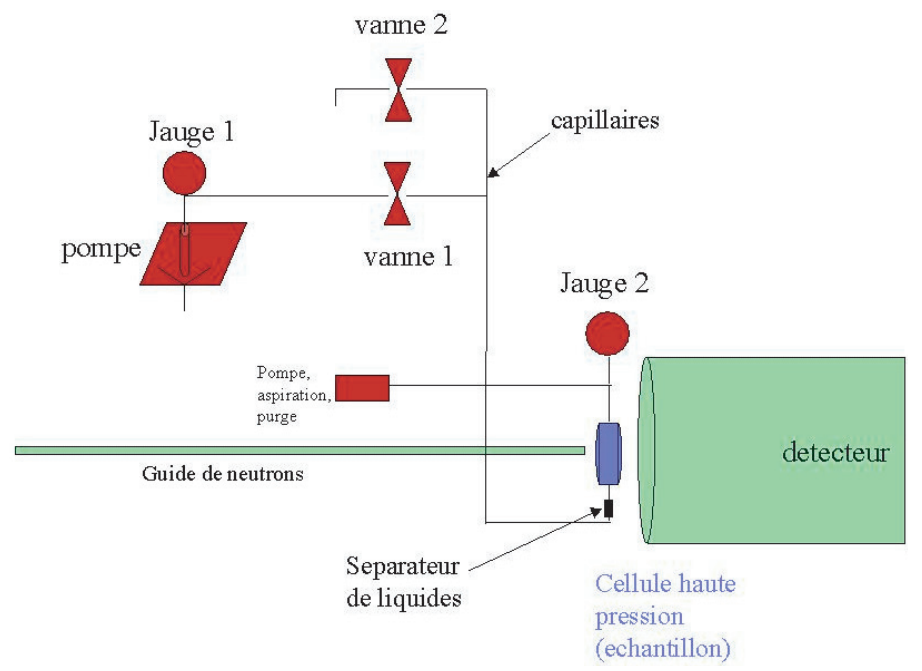

Figure 4. Montage expérimental pour le saut de pression et les mesures en SANS.

L'acquisition de données s'effectue par tranche de temps bien determinées, et à chaque cycle les données de la même tranche sont additionées afin d'augmenter la statistique.

Le montage actuel permet de monter à $2 \mathrm{kbars}$ dans la cellule pression, et d'effectuer les sauts de pression en $100 \mathrm{~ms}$. L'acquisition est declanchée par un signal donné par le capteur de pression, et commence exactement lorsque la pression atteint 2 kbars dans l'échantillon pour le saut $>0$, et 400 bars pour le saut $<0$. 


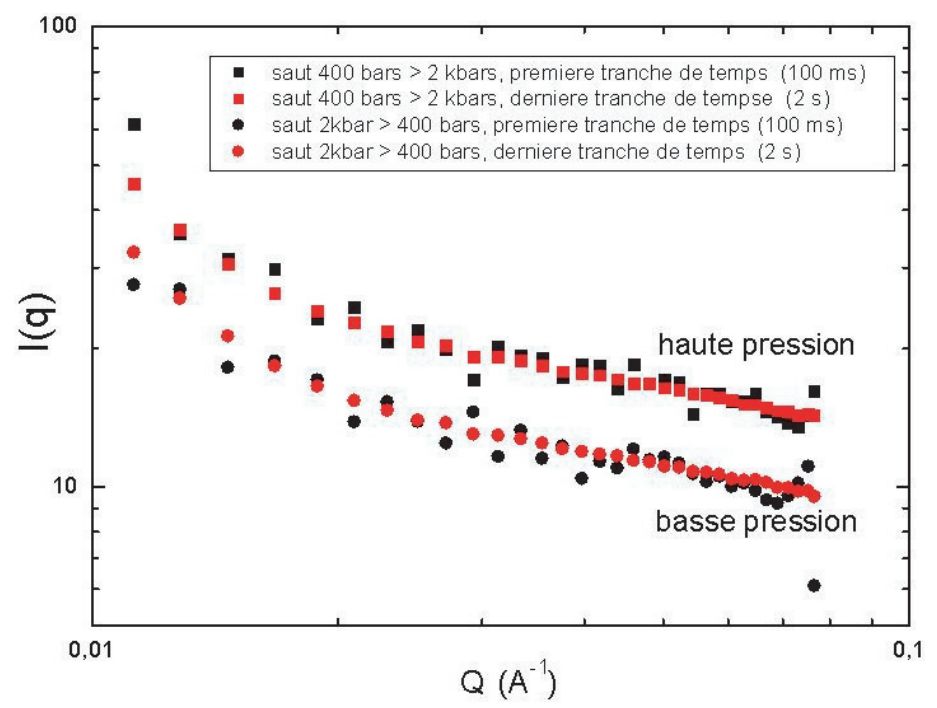

Figure 5. Exemple de données mesurées sur l' $\alpha$-lactalbumin à pH 7.0. Saut $>0$ et $<0$, juste après le saut de pression (noir) et quelques secondes plus tard (rouge). Les données sont moyennées sur une trentaine de cycles. Dans les deux sens de variation de la pression, la cinétique est plus rapide que $100 \mathrm{~ms}$.

\section{PREMIERS ESSAIS}

Des premiers tests ont étés réalisés avec le montage. Les mesures en cinétiques peuvent effectivement être effectuées, en moyennant sur plusieur dizaines de cycle. La figure 5 présente des données obtenues par (de)-pressurisation de l'alpha lactalbumin entre 400 bars et 2 kbars. Dans ce cas, la cinétique est plus rapide que $100 \mathrm{~ms}$.

\section{Références}

[1] Smeller L., Biochem. and Biophys. Acta 1595 (2002) 11-29; et articles du même volume.

[2] Segel D.J., Bachmann A., Hofrichter J., Hodgson K.O., Doniach S. and Kiefhaber T., J. Mol. Biol. 288 (1999) 489-499.

[3] Woenckhaus J., Kohling R., Winter R., Thiyagarajan P. and Finet S., Rev. of Sci. Inst. 71(10) (2000) 3895-3899. 